\title{
The Clinical Study of a New Management Model Using Mobile Health for Senile Hypertension
}

\author{
Jian Shi, Ping Wang, Aimin Wang* \\ Department of Health Management, Qingdao Municipal Hospital, Qingdao, China \\ Email: *dsong55cn@yahoo.com.cn
}

How to cite this paper: Shi, J., Wang, P. and Wang, A.M. (2019) The Clinical Study of a New Management Model Using Mobile Health for Senile Hypertension. Health, 11, 1087-1094.

https://doi.org/10.4236/health.2019.118085

Received: July 24, 2019

Accepted: August 20, 2019

Published: August 23, 2019

Copyright ( 2019 by author(s) and Scientific Research Publishing Inc. This work is licensed under the Creative Commons Attribution International License (CC BY 4.0).

http://creativecommons.org/licenses/by/4.0/

(c) (i) Open Access

\section{Abstract \\ Objective: To evaluate a new management model using mobile health for se-} nile hypertension. Methods: This medical service combined traditional medical treatment with Mobile Health. We use it to explore a new and effective model of elderly hypertension management and the most effective and lowest cost management crowd. According to the randomized controlled design of trial, 105 old hypertensive patients participated in the study voluntarily in the Qingdao Municipal Hospital were randomly divided into the experimental group ( 75 cases) and control group (30 cases). Experimental group is divided into geriatric specialist group ( 25 cases), general practitioner group ( 25 cases) and nurse group (25 cases). Blood pressure was administered in experimental (with the new model) and control groups (with the traditional model) for 2 months to compare their blood pressure and the decrease of them. Results: Blood pressure was compared between the two groups before and after administration. The systolic blood pressure (SBP) of experimental group is $162.44 \pm 13.970 \mathrm{mmHg}$ before administration. SBP of experimental group is $147.69 \pm 13.509 \mathrm{mmHg}$ after administration, the difference is statistically significant $(\mathrm{P}=0.000)$. The diastolic blood pressure (DBP) of experimental group is $91.58 \pm 10.822 \mathrm{mmHg}$ before administration. DBP of experimental group is $84.64 \pm 10.412 \mathrm{mmHg}$ after administration, $\mathrm{P}=0.003$. SBP of control group is $159.93 \pm 15.238 \mathrm{mmHg}$ before administration. The systolic blood pressure of control group is $152.33 \pm 14.115(\mathrm{mmHg})$ after administration. $\mathrm{P}$ is $0.167(\mathrm{P}>0.05)$. The difference was not statistically significant. DBP of control group is $94.73 \pm 12.401(\mathrm{mmHg})$ before administration. DBP of control group is $90.60 \pm 11.115(\mathrm{mmHg})$ after administration. $\mathrm{P}$ is $0.345(\mathrm{P}>$ $0.05)$. The difference was not statistically significant. The decrease of SBP of geriatric specialist group, general practitioner group and nurse group is 18.40 $\pm 11.764 \mathrm{mmHg}, 14.47 \pm 9.44 \mathrm{mmHg}, 10.80 \pm 6.026 \mathrm{mmHg}$. The difference was not statistically significant $(\mathrm{P}>0.05)$. The decrease of DBP of geriatric specialist group, general practitioner group and nurse group is $9.33 \pm 7.451$ 
$\mathrm{mmHg}$ vs. $5.33 \pm 6.287 \mathrm{mmHg}$ vs. $6.13 \pm 5.069 \mathrm{mmHg}(\mathrm{P}>0.05)$. Conclusion: The new model is effective and it works well for controlling blood pressure of elderly hypertensive patients. Especially with the doctors and patients' families, the management effect has been obviously improved. Medical interventions make the new model more scientific. The management of geriatric specialists, general practitioners and nurses is equally valid.

\section{Keywords}

Senile Hypertension, Management Model, Mobile Health

\section{Introduction}

The rapid development of intelligent wearable devices and intelligent terminals has brought the management of chronic diseases into the era of mobile medical care. The WTO defines mobile medical care as mobile Internet devices, such as mobile phones, computers and body movement monitoring, which also includes electronic medical records, remote consultation and various terminal monitoring devices. Mobile medicine extends the medical service and technology, combines the traditional medical and health service with the new Internet technology, improves the quality of medical and health service and guarantees the effect of medical service through the utilization of information resources. At present, China's health management and mobile medical mode are still not mature and in the stage of development discussion. In addition, the medical resource allocation in various regions is unbalanced and unreasonable, which is not compatible with the modern medical mode. This study discusses the effectiveness and feasibility of the "new mode of mobile platform management" for community hypertension management in the elderly.

\section{Objects and Methods}

\subsection{Objects}

Selection of Qingdao municipal hospital as the research point, according to a randomized controlled trial design, into the Qingdao municipal hospital voluntarily participate in the study of senile hypertension patients, 105 were randomly divided into experimental group 75 cases (average age $69.31 \pm 5.42$ years) and control group 30 cases (average age $71.13 \pm 6.39$ ), the experimental group is divided into the geriatrics specialist group of 25 cases, the general practitioner (GP) group of 25 cases, the nurse group of 25 people. There was no statistical difference in age and gender between the experimental group and the control group, which was comparable.

\subsection{The Diagnosis, Inclusion and Exclusion Criteria of Hypertension}

We accord to the diagnosis and classification criteria in the latest Chinese guide- 
lines for hypertension [1]. Inclusion criteria: patients with hypertension aged $>$ 60 years. Elimination criteria: 1 year have a stroke, myocardial infarction, such as cardiovascular emergency patients, secondary hypertension patients, patients with hypertensive crisis, and a history of malignant tumor and in the past in patients undergoing radiotherapy or chemotherapy, patients with mental disorder, patients with poor compliance, nearly a month in other related or not related researchers.

\subsection{Monitoring Equipment and Management Platform}

The remote intelligent sphygmomanometer used in this study is iHealth BP3 intelligent sphygmomanometer, which is suitable for IOS or Android devices. In this study, mobile medical apps used for remote blood pressure management were iHealth MyVitals (used by patients) and iHealth Pro (used by managers). WeChat public platform used in the doctor-patient communication process is "elderly HBP management platform".

\subsection{Control Group Management Methods}

The patients were given the traditional management of hypertension, the appropriate drug antihypertensive measurement, the reasonable daily education of hypertension, the knowledge of hypertension and the reasonable lifestyle, but the patients were not given the follow-up intervention for blood pressure control (Figure 1).

\subsection{Experimental Group Management Method}

Patients were given a "new mode of mobile platform management" to manage their blood pressure control. One geriatrician, one general practitioner and one nurse were trained to manage the specialist, general practitioners and nurses respectively. Establish a public platform for senior hypertension management. Managers (i.e., uniformly trained specialists, general practitioners and nurses)

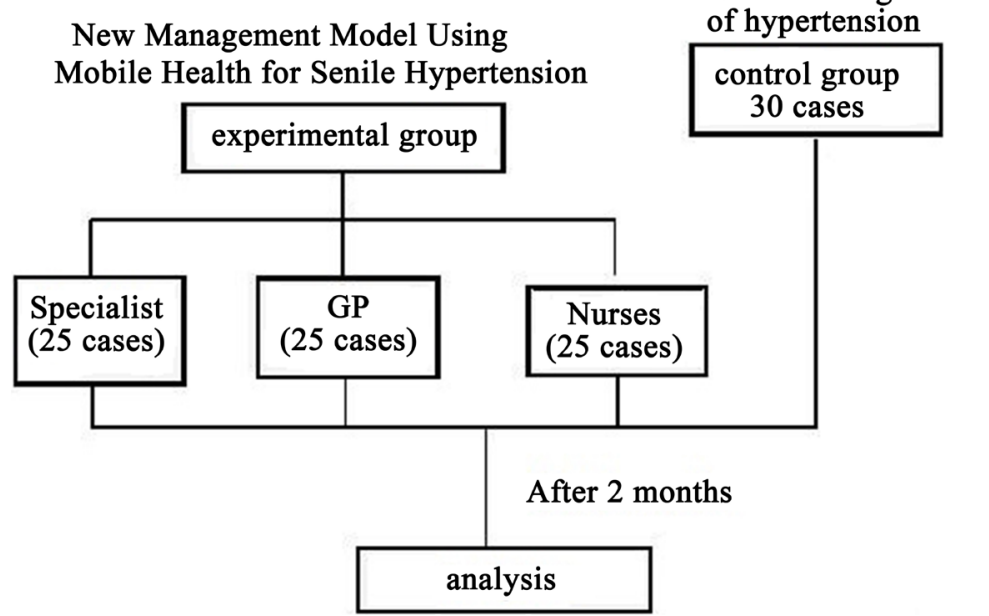

Figure 1. Flow chart of experimental method. 
provide health education, medication guidance and reasonable lifestyle guidance (diet and exercise, etc.) to the patients in this group through the public platform. Patients upload their blood pressure to the network through iHealth BP3 intelligent sphygometer, and communicate their problems with their own managers through the public platform. The managers monitor the blood pressure situation of the group of patients through the network and provide targeted individualized guidance. 1 - 2 times a month, including etiology, clinical manifestations and treatment.

Emphasized the importance of patients with hypertension of long-range take medicine, and instruct patients to take their medications regularly, inform patients lose weight, try to control the BMI $24 \mathrm{~kg} / \mathrm{m}^{2}$, reducing sodium intake, daily should not be more than $6 \mathrm{~g}$, reduce fat intake, supplement Sylvie, eat fresh vegetable and fruit more, give up smoking, drinking adequate exercise, adjust the way of life, folic acid preparation when necessary. According to the fluctuation of blood pressure of the patients, the types and dosage of antihypertensive drugs were adjusted, the appropriate time of taking the drugs was mastered, and follow-up observation was strengthened within one week after the drug adjustment.

All the patients included in the study underwent comprehensive intervention for 2 months, and the main observation indexes included systolic blood pressure, diastolic blood pressure and blood pressure fluctuation values of the patients.

\subsection{Statistical Methods}

PASWStatistics18 software was used to establish database and statistical analysis; mean standard deviation was used for parameter continuous variables; $t$ test was used for two groups; Analysis of variance was used to compare the decreased blood pressure values of the three subgroups in the experimental group, and LSD-t test was used for further pairwise comparison; Variance analysis was used to compare continuous variables among groups. $\mathrm{P}<0.05$ was considered statistically significant.

\section{Results}

1) Baseline data analysis: The total number of experimental samples is 106 , among which there are 42 males and 33 females in the experimental group and 20 males and 10 females in the 30 control group. There was no statistically significant difference between the experimental group and the control group in age, gender, smoking history, diabetes mellitus, blood pressure classification, and types of antihypertensive drugs, suggesting that the experimental group and the control group were comparable (Table 1).

2) Before and after intervention were compared and analyzed: After 2 months of intervention, the experimental group adopted the new management mode of mobile platform management, while the control group adopted the tra- 
ditional management mode. Blood pressure values of the two groups before and after intervention were shown in Table 2. Systolic blood pressure of the experimental group was $162.44 \pm 13.970(\mathrm{mmHg})$ before intervention, and $147.69 \pm$ $13.509 \mathrm{mmHg}$ after intervention, $\mathrm{P}=0.000$, with statistically significant differences. The diastolic blood pressure before intervention was $91.58 \pm 10.822$ $\mathrm{mmHg}$, and the systolic blood pressure after intervention was $84.64 \pm 10.412$ $\mathrm{mmHg}, \mathrm{P}=0.003$, indicating statistically significant differences. Systolic blood pressure of the control group was $159.93 \pm 15.238 \mathrm{mmHg}$ before intervention and $152.33 \pm 14.115 \mathrm{mmHg}$ after intervention, $\mathrm{P}=0.167$, with no statistically significant difference. The diastolic blood pressure of the control group was $94.73 \pm 12.401 \mathrm{mmHg}$ before intervention and $90.60 \pm 11.115 \mathrm{mmHg}$ after intervention, $\mathrm{P}=0.345(\mathrm{P}>0.05)$, with no statistically significant difference.

3) Comparison and analysis of blood pressure drop in subgroup before and after intervention: After 2 months of intervention, the three subgroups of the experimental group were geriatrician group, general practitioner group and nurse group, respectively. The blood pressure drop values were shown in Table 3.

Table 1. Comparison of general clinical data between the two groups.

\begin{tabular}{cccc}
\hline Clinical parameters & $\begin{array}{c}\text { Control group } \\
(30 \text { cases })\end{array}$ & $\begin{array}{c}\text { Experimental group } \\
(75 \text { cases })\end{array}$ & $P$ \\
M/F (case) & $20 / 10$ & $42 / 33$ & 0.285 \\
Age $(\bar{x} \pm \mathrm{s}, \mathrm{y})$ & $71.13 \pm 6.39$ & $69.31 \pm 5.418$ & 0.450 \\
Smoke (Y/N) & $18 / 12$ & $42 / 33$ & 0.104 \\
Diabetes (Y/N) & $14 / 16$ & $18 / 57$ & 0.178 \\
$\begin{array}{c}\text { Blood pressure levels } \\
(1 \text { and 2 Level/3 Level) }\end{array}$ & $18 / 12$ & $58 / 17$ & 0.247 \\
$\begin{array}{c}\text { Anti-hypertension drugs } \\
(\leq 2 />2 \text { kinds })\end{array}$ & $9 / 6$ & $34 / 11$ & \\
\hline
\end{tabular}

Table 2. Before and after intervention were compared within group ( $\mathrm{mmHg}$ ).

\begin{tabular}{|c|c|c|c|c|c|c|}
\hline & \multicolumn{3}{|c|}{ Control group } & \multicolumn{3}{|c|}{ Experimental group } \\
\hline & Before & After & $\mathrm{P}$ & Before & After & $\mathrm{P}$ \\
\hline SBP & $162.24 \pm 13.67$ & $147.69 \pm 13.51$ & 0.000 & $159.93 \pm 15.24$ & $152.33 \pm 14.12$ & 0.167 \\
\hline DBP & $91.58 \pm 10.82$ & $84.64 \pm 10.41$ & 0.003 & $94.73 \pm 12.40$ & $90.60 \pm 11.12$ & 0.345 \\
\hline
\end{tabular}

Systolic blood pressure: SBP; diastolic blood pressure: DBP.

Table 3. Decreased values of blood pressure were compared before and after intervention in experimental group.

\begin{tabular}{lccccc}
\hline & Specialist group & GP group & Nurses group & $F$ & P \\
\hline Decreased value SBP (mmHg) & $18.40 \pm 11.764$ & $14.47 \pm 9.441^{\mathrm{a}}$ & $10.80 \pm 6.026^{\mathrm{a}, \mathrm{b}}$ & 2.464 & 0.097 \\
Decreased value DBP (mmHg) & $9.33 \pm 7.451$ & $5.33 \pm 6.287^{\mathrm{a}}$ & $6.13 \pm 5.069^{\mathrm{a}, \mathrm{b}}$ & 1.670 & 0.201 \\
\hline
\end{tabular}

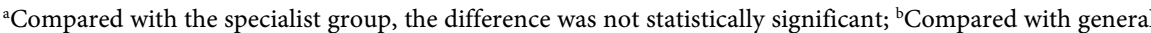
practitioners group, the difference was not statistically significant. 
Their systolic blood pressure decreased by $18.40 \pm 11.764 \mathrm{mmHg}, 14.47 \pm 9.441$ $\mathrm{mmHg}$ and $10.80 \pm 6.026 \mathrm{mmHg}$, respectively, $\mathrm{P}=0.097(\mathrm{P}>0.05)$, showing no statistically significant differences. Diastolic blood pressure decreased by $9.33 \pm$ $7.451 \mathrm{mmHg}, 5.33 \pm 6.287 \mathrm{mmHg}$ and $6.13 \pm 5.069 \mathrm{mmHg}$, respectively, $\mathrm{P}=$ $0.201(\mathrm{P}>0.05)$, showing no statistically significant differences.

\section{Discussion}

This experiment confirms the scientific effectiveness of the "new mode of mobile platform management". Meanwhile, the randomized control evaluation shows that geriatricians, general practitioners and nurses are equally effective in management, and there is no significant difference in the management process and management effect among the management groups. It makes full use of the combination of medical intervention and Internet mobile platform to improve the remote and real-time management effect of elderly hypertension, especially with the joint participation of medical staff and patients' families, greatly improving the reduction of blood pressure and blood pressure fluctuation value.

"New mode of mobile platform management" is effective in the management of elderly hypertension, which not only reduces the blood pressure of patients, but also reduces the fluctuation value of patients' blood pressure, and improves the compliance rate of blood pressure, the level of patient self-management and satisfaction. The results showed that SBP and DBP in the experimental group were lower after intervention than before intervention, and the difference was statistically significant $(\mathrm{P}<0.05)$. The SBP and DBP values in the control group showed no significant difference from those before the intervention. This shows that "new model" mobile platform management of elderly hypertension is feasible and effective, not only reduces the blood pressure value of the elderly patients and also reduces the patient's blood pressure fluctuation at the same time, greatly reduced due to blood pressure fluctuations caused by the damage of important organs, is a new kind of mode, management of elderly hypertension can help control blood pressure, slow down the progress of the disease. Based on the problem of old age-old blood pressure management in our country and national conditions, "new model" mobile platform management can increase elderly patients for the cognition of hypertension, the right to recognize the harmfulness of hypertension, although the clinical manifestations of the disease are easily ignored by us, the elderly tend to embrace "no sick is not sick" wrong ideas, leading to a high incidence of various complications of hypertension, eventually leading to malignant events happened.

New model "and" mobile platform management of elderly hypertension can save medical resources, manage hypertension patients by mobile medical network. For patients at home, most of the time management is self-management, which greatly reduces the burden of the community and hospital, sees a doctor to ensure the feasibility of basic health service, for the need of patients in a timely manner to enjoy medical resources has made a contribution, at the same time 
also promoted the family members together to participate in the management and supervision, increase the communication to the patients' families, but also indirectly reduce the medical burden of the family, the patient's trust between family members, to improve the compliance of elderly patients. Elderly patients and their families can know patients' conditions through mobile medical APP and WeChat public platform, and communicate with medical staff directly, which greatly improves patients' and their families' control of diseases and their initiative in disease management. Meanwhile, it can also increase doctor-patient communication, relieve the tension between doctors and patients caused by various social parties, and increase the satisfaction of patients [2]. Using the mobile medical model to manage hypertension in the elderly can also increase the sense of participation of the elderly, reduce their loneliness, and alleviate the increasing status of functional diseases in the elderly.

In the management of geriatric diseases in China, most patients, especially those with chronic senile diseases, rely on the community for their disease management, and most of their managers are general practitioners. In this study, SBP and DBP were lower in geriatrician group, general practitioner group and nurse group after intervention than before intervention, but there was no statistically significant difference $(\mathrm{P}>0.05)$. This shows that geriatricians, general practitioners and nurses are equally effective in the management of hypertension in the elderly, and there is no significant difference in the management process and management effect among different management groups.

At present more popular on the blood pressure monitoring and management of hypertension in the application, only a small part by universities, hospitals, or related to hypertension, most applications are developed by the third party company, in this case, people will pay more attention to the application of scientific and accurate [3]. Medical intervention adds science and accuracy to the "new mode of mobile platform management". Because thousands of apps are available in stores, most of them come with a generic "fitness" or "wellness" label, which is often "not for medical purposes" [4]. Only a few are certified by experts in the relevant fields, with high accuracy and credibility, as well as the privacy and security of user data. Because all of these key issues will become important issues for public health and for individual patients seeking care.

Looking forward to the application of mobile medical treatment in the elderly hypertension management, the current health management and mobile medical model in China is still not mature, in the development of the discussion stage, coupled with the distribution of medical resources around the imbalance, unreasonable, there is a phenomenon that does not adapt to the contemporary medical model [5]. There are still some shortcomings in mobile medicine, such as the younger user group. The new mode of using the Internet for medical treatment is still more accepted by young people. With the exception of a few diseases, more diseases, especially chronic diseases, are more common among the elderly, who are still less receptive to $\mathrm{m}$-health care. Problem solving is high- 
ly biased. When using mobile medical care for medical treatment, the form is more inclined to ask for medical treatment, and doctors have great restrictions on the physical examination of patients, so most online patients can only consult, while the real diagnosis still needs to be confirmed offline in hospital. Patients face hidden dangers of information leakage [6].

With the rapid development of mobile healthcare, as an emerging industry, the standardized management system of this industry is not comprehensive, and various industries, companies and individuals are flooding into this industry, among which there are some hidden risks that lead to the disclosure of patient information driven by some interests [7]. The "new mode of mobile platform management" designed in this experiment has also encountered many obstacles in the implementation of the management of hypertension in the elderly. A small number of elderly patients are able to skillfully use mobile medical apps. Most of them are unfamiliar with smart phones and the Internet, and have poor ability to accept new things, which greatly hinders the experiment.

The "New mode of mobile platform management" is feasible to manage hypertension in the elderly, especially with the joint participation of medical staff and patients' families; the management effect is significantly proved. Compared with the traditional hypertension management model, it is more suitable for elderly hypertension patients. The combination of medical intervention and "new mode of mobile platform management" makes the management mode more rational and scientific.

\section{Conflicts of Interest}

The authors declare no conflicts of interest regarding the publication of this paper.

\section{References}

[1] (2019) Chinese Guidelines for the Prevention and Treatment of Hypertension (Revised 2018). Chinese Journal of Cardiovascular Medicine, 1.

[2] Zhang, F. (2017) Discussion on Self-Management Model of Elderly Hypertension Patients in Community by Higher Vocational Nursing Students. Health Vocational Education, 12, 78-79.

[3] Zhang, J.Y., Ren, Z.F. and Liu, B. (2015) Construction Idea of Intelligent Prevention and Control Platform for Hypertension. Journal of China-Japan Friendship Hospital, 2, 120-122.

[4] Li, L. and Yuan, X.H. (2014) Management of Chronic Diseases in Urban Community. Chinese Health Quality Management, 3, 108-110.

[5] Cui, T., Gong, Q.J. and Li, F. (2014) Effects of Chronic Disease Management Model on Elderly Hypertension Patients in a Community. Chinese Journal of Convalescent Medicine, 10, 931-933.

[6] (2006) Guidance of the State Council on Developing Urban Community Health Services. Chinese General Practice, 5, 349-350.

[7] Liu, Y.X. (2017) Effect Analysis of Mobile Medical Service Mode for Hypertension and Diabetes. Drug Evaluation, 11, 15-17. 